\title{
Selective extraction of aliphatic amines by functionalized mesoporous silica-coated solid phase microextraction Arrow
}

\author{
Hangzhen Lan ${ }^{1,2} \cdot$ Wenzhong Zhang ${ }^{1} \cdot$ Jan-Henrik Smått ${ }^{3} \cdot$ Risto T. Koivula $^{1} \cdot$ Kari Hartonen $^{1,2}$ • \\ Marja-Liisa Riekkola ${ }^{1,2}$ (B)
}

Received: 7 February 2019 / Accepted: 19 May 2019 / Published online: 11 June 2019

(C) The Author(s) 2019

\begin{abstract}
Mesoporous silica-coated solid phase microextraction (SPME) Arrow systems were developed for capturing of low-molecular-weight aliphatic amines (LMWAAs) from complicated sample matrices. Specifically, silicas of type MCM-41, SBA-15 and KIT-6 were chosen as substrates to afford size-exclusion selectivity. They possess ordered multidimensional pore-channel structures and mesopore sizes between 3.8 and $8.2 \mathrm{~nm}$. Their surface acidity was enhanced by grafting them with a layer of titanium hydrogenphosphate (-TP). This enhanced the chemical selectivity for basic LMWAAs. The siliceous coatings increased the extraction of ethylamine, diethylamine (DEA) and triethylamine (TEA) by factors of 18.6-102.5, 4.8-10.8 and 2.6-4.0, respectively, when compared to the commercial SPME Arrow with polydimethylsiloxane/divinylbenzene coating. Among them, the MCM-41 and MCM-41-TP coated SPME Arrows demonstrated exceptional selectivity towards LMWAAs that were quantified by gas chromatography-mass spectrometry (GC-MS). The total peak area ratios of LMWAAs/ten competing compounds were 25.4 and 36.3, respectively. The extraction equilibrium was reached within 20-30 min. The MCM-41 and MCM-41-TP derived SPME Arrows gave very similar results (18.4 $\pm 2.1-376 \pm 12 \mathrm{ng} \mathrm{g}^{-1}$ to DEA and TEA) when applied to urban mushroom samples. SPME Arrow with MCM-41 coatings followed by GC-MS was applied also to the analysis of atmospheric air and urine samples resulting in high selectivity due to the size and mesoporous structure of the functionalized silica, and its chemical interactions with the LMWAAs.
\end{abstract}

Keywords Functionalized mesoporous silica material - Solid phase microextraction Arrow - Selective extraction · Low-molecular-weight aliphatic amines $\cdot$ Gas chromatography-mass spectrometry $\cdot$ Mushroom $\cdot$ Atmospheric air $\cdot$ Urine

\section{Introduction}

Low-molecular-weight $\mathrm{C}_{1}$ - $\mathrm{C}_{6}$ aliphatic amines (LMWAAs) play a vital role in atmospheric and physiological processes. In the Earth's atmosphere, LMWAAs lead to the formation of

Electronic supplementary material The online version of this article (https://doi.org/10.1007/s00604-019-3523-5) contains supplementary material, which is available to authorized users.

Marja-Liisa Riekkola

marja-liisa.riekkola@helsinki.fi

1 Department of Chemistry, University of Helsinki, P.O. Box 55, 00014 Helsinki, Finland

2 Institute for Atmospheric and Earth System Research, University of Helsinki, P.O. Box 64, 00014 Helsinki, Finland

3 Laboratory of Physical Chemistry, Åbo Akademi University, Porthansgatan 3-5, 20500 Turku, Finland salt particles and nitrogen-containing organics and thereby eventually contribute to the nucleation and growth of secondary organic aerosols (SOAs) [1-3]. Natural vegetation constitutes one of the most widespread biosphere sources of the airborne amines $[1,4,5]$. Quantitation of LMWAAs emission from remote regions is therefore essential to help underpin the mechanisms of aerosol formation and growth process. In physiological processes, the breakdown of foodborne choline and lecithin by intestinal microbes produces endogenous LMWAAs [6-8]. However, disruptions on normal hepatic or renal activities cause LMWAAs accumulation in the bloodstream $[6,9]$, elevating the risk of forming carcinogens (i.e. nitrosamines) and neurotoxins (diethylamine (DEA)) [7, 9, 10]. Hence, early-stage disease diagnosis aided by metabolite profiling benefits from the accurate measurement of urinary LMWAAs concentration.

The measurement of low-concentration $\left(<\mathrm{ng} \mathrm{mL}^{-1}\right)$ LMWAAs from complex matrices is not a straightforward task. They are intrinsically highly volatile, polar, reactive, 
and water soluble. Therefore, their selective and rapid adsorption and enrichment is necessary prior to instrumental analysis. Solid phase microextraction (SPME) Arrow is a simple, time-efficient, robust and reliable sample pretreatment technique that has become more popular just in the past few years [11-15]. In order to achieve targeted separation of LMWAAs, the adsorbent hosted on SPME Arrow needs to be tailored.

Engineered porous materials are widely employed for selective gas adsorption and separation. The selectivity arises synergistically from the synthetic control over the pore-channel systems as well as the surface chemical properties. For gas molecules, micro- and mesoporous materials provide excellent shape recognition. To this end, metal organic frameworks (MOFs), porous carbonbased materials and ordered mesoporous silica, have been designed and used as selective adsorbents due to their tailorable structure, tunable pore geometry, high specific surface area and good porosity $[16,17]$. In our previous work, although mesoporous acidified zeolite imidazolate framework-8 (ZIF-8) and microporous Carboxen 1000 coated SPME Arrows demonstrated satisfactory extraction selectivity towards LMWAAs in atmospheric air, wastewater, salmon and mushroom samples $[12,14]$, MOFs had a limited pore accessibility for analytes [14]. Porous carbon-based materials feature an ultrahigh thermal and chemical stability, while difficulties are met in their surface functionalization [18-21]. Mesoporous silica materials offer more flexibility for pore sizes (from a few up to dozens of nanometers) [22] and they have better chemical and thermal stability than MOFs. Further, it is easier to functionalize their mesoporous inner walls compared to those of carbon materials [22]. However, the most promising characteristic of mesoporous silica is its natural acidic surface that predicts a high affinity to basic amines. For all abovementioned merits, ordered mesoporous silica seems to be a good alternative to other classical adsorbents for LMWAAs extraction. Additionally, chemical modification of the mesoporous silica with appropriate functional groups can improve their extraction selectivity towards LMWAAs.

The purpose of this study was to construct mesoporous silica materials for targeted and fast extraction of LMWAAs from different sample matrices. Six ordered mesoporous silica materials with various pore sizes, pore structures and surface functionalities were synthesized and then individually fabricated on SPME Arrow system. Extraction selectivity of the mesoporous silica materials for LMWAAs was clarified, at under $1 \%$ and $50 \%$ humidity levels, and compared with that of commercial adsorbents. The applicability of SPME Arrow system including our new materials was further tested by exploiting them for detection of LMWAAs in mushroom, atmospheric air and urine samples.

\section{Experimental}

\section{Reagents and materials}

Triethylamine (TEA) (99\%), aniline (>99.5\%), dimethylformamide (DMF) (99.9\%) and polyacrylonitrile (PAN) $(\mathrm{Mw}=150,000)$ were purchased from Sigma-Aldrich (St. Louis, USA); ethanol (100\%) and toluene (HPLC grade) were from VWR Chemicals (Pennsylvania, USA); ethylamine (EA) (70\% in water), benzyl alcohol (99.5\%), diethylamine (DEA) $(\geq 99.7 \%)$, benzyl acetate $(\geq 99 \%)$ and decane $(>99 \%)$ were from Fluka (The Netherlands); acetone $(\geq 99.8 \%)$, tetrahydrofuran $(\geq 99.9 \%)$ and ethyl acetate $(\geq 99.7 \%)$ were from Honeywell (Honeywell GmbH, Seelze, Germany); 2pentylfuran (98\%) was from Alfa Aesar (Karlsruhe, Germany); and acetophenone was from The British Drug Houses Ltd. (Poole, England).

Tetraethyl orthosilicate $(98 \%)$, hexadecyltrimethylammonium bromide $(>99.0 \%)$, poly(ethylene oxide)-block-poly(propylene oxide)-block-poly(ethylene oxide) with an average molecular weight of 5800, and 1-butanol (>99.7\%) were used for silica synthesis. Titanium (IV) isopropoxide $\left(\mathrm{Ti}\left(\mathrm{OPr}^{i}\right)_{4}\right)(>97 \%)$ and phosphorus oxychloride $(>99 \%)$ were used for functionalization. All of them were from Sigma-Aldrich.

Polydimethylsiloxane (PDMS), PDMS-Carboxen1000, PDMS-divinylbenzene (DVB) and Carboxen WR SPME Arrows (sorbent film thickness $120 \mu \mathrm{m}$ and the sorbent length $20 \mathrm{~mm}$ ) and PAL RTC auto-sampler were kindly provided from CTC Analytics AG (Zwingen, Switzerland). Bare Arrows (for coating length of $20 \mathrm{~mm}$ ) were from BGB Analytik AG (Zurich, Switzerland).

\section{Materials synthesis}

The mesoporous MCM-41, SBA-15 and KIT-6 silica materials were synthesized via sol-gel template routes following the literature procedures (Fig. 1) [23-25]. The details are given in the Supporting Material.

The functionalization of the mesoporous silica with titanium phosphate moieties was conducted by surface grafting techniques [26]. The details are found from the Supporting Material.

Thus, totally six mesoporous silica materials were synthesized: 1) MCM-41, 2) SBA-15, 3) KIT-6, 4) titaniumphosphate modified MCM-41 (MCM-TP), 5) titaniumphosphate modified SBA-15 (SBA-TP), and 6) titaniumphosphate modified KIT-6 (KIT-TP).

\section{Fabrication of SPME Arrow coatings}

Firstly, $0.2 \mathrm{~g}$ of PAN was heated at $90^{\circ} \mathrm{C}$ in $10 \mathrm{~mL}$ of DMF for $1 \mathrm{~h}$ to form the binder solution. After cooling down to room 
Fig. 1 Synthesis route of functionalized silica materials including MCM-TP, SBA-TP and KIT-TP

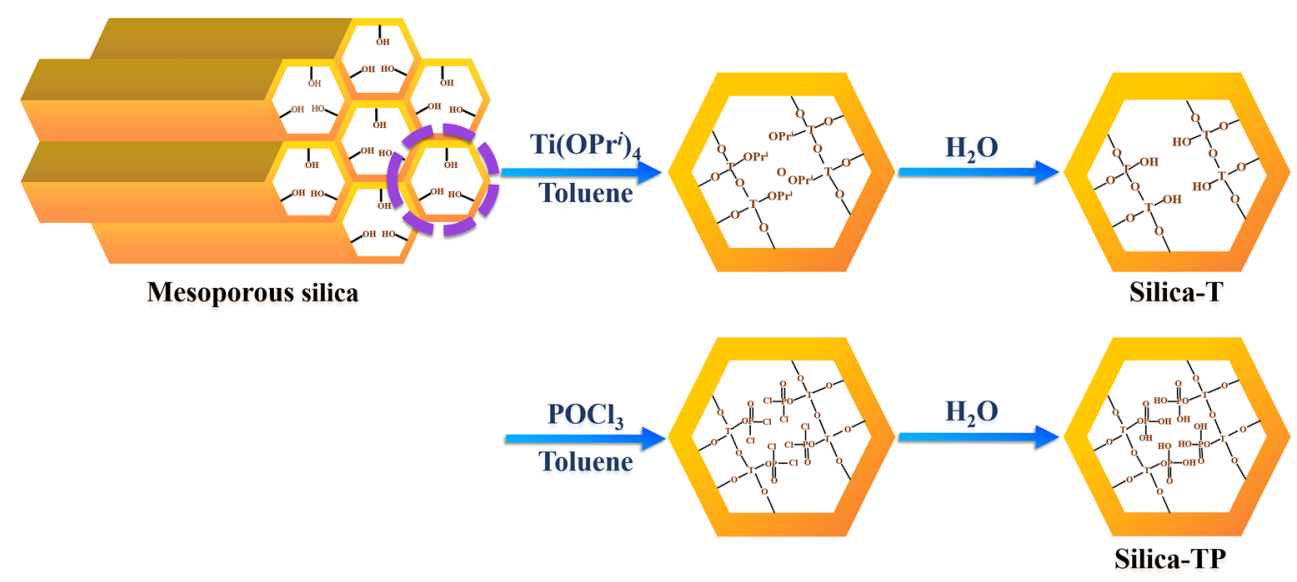

Arrow steps. Six laboratory-made and four commercial SPME Arrow coatings (PDMS, PDMS-DVB, PDMSCarboxen1000 and Carboxen WR) were compared in terms of extraction affinity and selectivity towards LMWAAs in dynamic SPME mode. The procedure was as follows: precleaning at $250{ }^{\circ} \mathrm{C}$ in the auto-sampler conditioner for $10 \mathrm{~min} ; 10 \mathrm{~min}$ extraction in the sampling port at room temperature $\left(\sim 22{ }^{\circ} \mathrm{C}\right)$; desorption at $250{ }^{\circ} \mathrm{C}$ in the $\mathrm{GC}$ inlet for $1 \mathrm{~min}$.

\section{SPME Arrow procedures for analytical method development and natural sample analysis}

SPME Arrows coated successfully with MCM-41- and MCM-TP were ready for the testing. To a $20 \mathrm{~mL}$ headspace vial, $5 \mathrm{~mL}$ of sample with $2 \mathrm{~g}$ of $\mathrm{NaCl}$ were placed and vial was closed with a PTFE/silicone septum screw-cap (both from Phenomenex, Torrance, California, USA). Then $250 \mu \mathrm{L}$ of $5 \mathrm{M} \mathrm{KOH}$ were injected through the septum with a syringe to release the amines into the headspace. The vial was incubated at $40^{\circ} \mathrm{C}$ for $15 \mathrm{~min}$. At the same time, SPME Arrow was pre-cleaned at $260{ }^{\circ} \mathrm{C}$ for $10 \mathrm{~min}$. Samples were extracted at $40{ }^{\circ} \mathrm{C}$ with $250 \mathrm{rpm}$ agitation for $20 \mathrm{~min}$ (MCM-41-SPME Arrow) or $30 \mathrm{~min}$ (MCM-TP-SPME Arrow). At the end, SPME Arrows were cleaned at $260{ }^{\circ} \mathrm{C}$ for $1 \mathrm{~min}$.

\section{Natural sample preparation}

\section{Mushroom sample preparation}

Six types of mushrooms (Figure S1) were collected from a forest near Kumpula Campus of the University of Helsinki (Helsinki, Finland) on the 8th of October, 2018. They were pretreated and analyzed during the same day without storage. Each sample ( $4 \mathrm{~g}$ ) was mixed with $10 \mathrm{~mL}$ of $10 \%$ formic acid $(v / v)$ and homogenized with a kitchen blender (Bosch, Gerlingen, Germany) for $10 \mathrm{~min}$. The homogenate was centrifuged three times at $5000 \times g$ for $15 \mathrm{~min}$. The supernatants
A PAL Cycle Composer (CTC analytics) software and a PAL RTC auto-sampler controlled and performed all the SPME

\section{On-line dynamic SPME Arrow procedures for coating comparison}


were combined and adjusted to the final volume of $100 \mathrm{~mL}$ with $10 \%$ formic acid $(\mathrm{v} / \mathrm{v})$.

\section{Atmospheric air sample preparation}

Atmospheric air samples were collected at SMEAR II (Station for Measuring Ecosystem-Atmosphere Relations II) Station in a Scots pine forest at Hyytiälä in southern Finland [27]. The sampling site was located about $15 \mathrm{~m}$ from a $127-\mathrm{m}$ high mast for atmospheric and flux measurements $\left(61^{\circ} 50^{\prime} 50.55^{\prime \prime} \mathrm{N}\right.$, $24^{\circ} 17^{\prime} 39.77^{\prime \prime} \mathrm{E}, 181 \mathrm{~m}$ above the sea level). The forest in Hyytiälä is around 50 years old and dominated by Scots pine, Norway spruce, birch and European aspen. The predominant plant species at the ground level are lingonberry, bilberry, wavy harigrass and heather and the most common mosses are Schreber's big red stem moss and a dicranum moss [4].

The sampling was done from the 23rd to 26th of October, 2018. The Denuder sampler for air located at $2 \mathrm{~m}$ above the ground vegetation and $3 \mathrm{~m}$ from the closest tree. A threechannel annular Denuder (242 mm length, Teflon-coated, stainless steel sheath, URG, Chapel Hill, USA) coated with $0.1 \mathrm{M}$ phosphoric acid was connected to a peristaltic pump with a flow rate of $1 \mathrm{~m}^{3} \mathrm{~h}^{-1}$. The sampling times ranged from 4 to $13.5 \mathrm{~h}$. After sampling, the Denuder was rinsed three times with $5 \mathrm{~mL}$ of Milli-Q water and the eluent was stored at $4{ }^{\circ} \mathrm{C}$ for further analysis. Totally 12 samples were collected.

\section{Urine sample}

Urine sample was collected from a young male volunteer (28 years old) without additional pretreatment.

\section{Instrumentation and GC-MS analysis}

An Agilent $6890 \mathrm{~N}$ gas chromatograph coupled with an Agilent 5975C mass selective detector (Agilent Technologies, Palo Alto, USA) was employed for the GCMS analysis. An InertCap ${ }^{\mathrm{TM}}$ for Amines capillary column (30 m length with $0.25 \mathrm{~mm}$ i.d., GL Sciences, Tokyo, Japan) was used for the chromatographic separations. The GC-MS conditions for analysis were as follow: oven temperature program: $40^{\circ} \mathrm{C}$ (held for $2 \mathrm{~min}$ ) and then increased to $250^{\circ} \mathrm{C}$ at a rate of $20^{\circ} \mathrm{C} \mathrm{min}^{-1}$ (held for $5 \mathrm{~min}$ ). Injector, transfer line, ion source and quadrupole temperature were 260, 250, 230 and $150{ }^{\circ} \mathrm{C}$; respectively. Electron ionization (EI) mode $(70 \mathrm{eV})$ was utilized $(m / z, 20-350)$. Helium (99.996\%, AGA, Espoo, Finland) was used as carrier gas at a constant flow rate of $1.2 \mathrm{~mL} \mathrm{~min}^{-1}$.

The surface morphologies of mesoporous silica materials were studied by scanning electron microscopy (SEM) (Hitachi, model S-4800, Japan). For their thermal stability measurements under nitrogen flow the thermogravimetric analysis (TGA) with a Mettler Toledo Star ${ }^{\mathrm{e}}$ system equipped with a TGA 850 thermobalance was used with the ramp rate of $10{ }^{\circ} \mathrm{C} \mathrm{min}^{-1}$. Small angle X-ray diffraction patterns were collected on a PANalytical X'Pert PW3710 MPD diffractometer with X-ray sourced from monochromatic $\mathrm{CuK} \alpha$ line $(\lambda=$ $1.54056 \AA$ ). The contents of Ti and P were determined on an Agilent microwave plasma-atomic emission spectrometer (MP-AES 4200) after a total dissolution in $65 \% \mathrm{HNO}_{3}$ and $1 \%$ HF using a CEM MARS 5 microwave digestion system. Nitrogen physisorption measurements were carried out on an Autosorb-1 instrument from Quantachrome Instruments. The samples were degassed at $200^{\circ} \mathrm{C}$ for $5 \mathrm{~h}$ prior to analysis. The BET surface area was determined in the pressure range of $0.05-0.20 \mathrm{P} / \mathrm{Po}$, while the pore size distribution was assessed using the NLDFT model for cylindrical pores.

\section{Results and discussion}

Six ordered mesoporous silica materials with different pore sizes and functional groups were synthesized and characterized with various methods. Then SPME Arrows coated with these materials were fabricated and compared with commercial SPME Arrows for selective extraction of amines at under $1 \%$ and $50 \%$ humidity levels. The parameters affecting the selectivity of mesoporous silica materials towards LMWAAs were studied. Two SPME Arrow-GC-MS methods were established by using the two most selective mesoporous silica materials for the identification and quantitation of trace amines in mushroom, atmospheric air and urine samples.

\section{Materials characterization}

In this work, three types of amorphous mesoporous silica materials were employed as substrates for the synthesis of adsorbents due to their flexible pore sizes and structures. MCM-41 and SBA-15 have two-dimensional (2D) hexagonal pore structures (Fig. 2a-b) with distinctive pore sizes ( $\sim 4$ vs $\sim 8 \mathrm{~nm}$ ) (Fig. 2d-e) [28, 29], and KIT-6 has a specific 3D cubic structure composed of two intertwined subnetworks (Fig. 2c) and approximately the same pore size as SBA-15 (Fig. 2f) [30]. After grafted with the -TP layer inside the wall, the average pore size and pore volume of silica decreased by $\sim 1 \mathrm{~nm}$ and $0.24-0.49 \mathrm{~cm}^{3} \mathrm{~g}^{-1}$, respectively (Fig. 2d-f). The results from small angle XRD (Fig. 2a-c) demonstrated that the (100) reflection of the mesoporous silica decreased after TP layer modification. This means that the modification resulted in ordered smaller pores, with some pore blockages. The layer-by-layer grafting technique of -TP layer to silica surface, described in literature [31] resulted in reproducible results. The nitrogen sorption-desorption and XRD data (Fig. 2) indicates that the -TP groups are preferentially grafted onto the mesopore walls rather than filling up the pores. Further, the grafted groups, $\sim 8-12$ wt. $\%$ of $-\mathrm{T}$ and $\sim 4-$ 
Fig. 2 Small-angle XRD patterns $\left(0.5-5^{\circ}\right)$ and density functional theory (DFT) pore size distribution of (a and d) MCM-41 series, (b and e) SBA-15 series, and (c and f) KIT-6 series silica materials
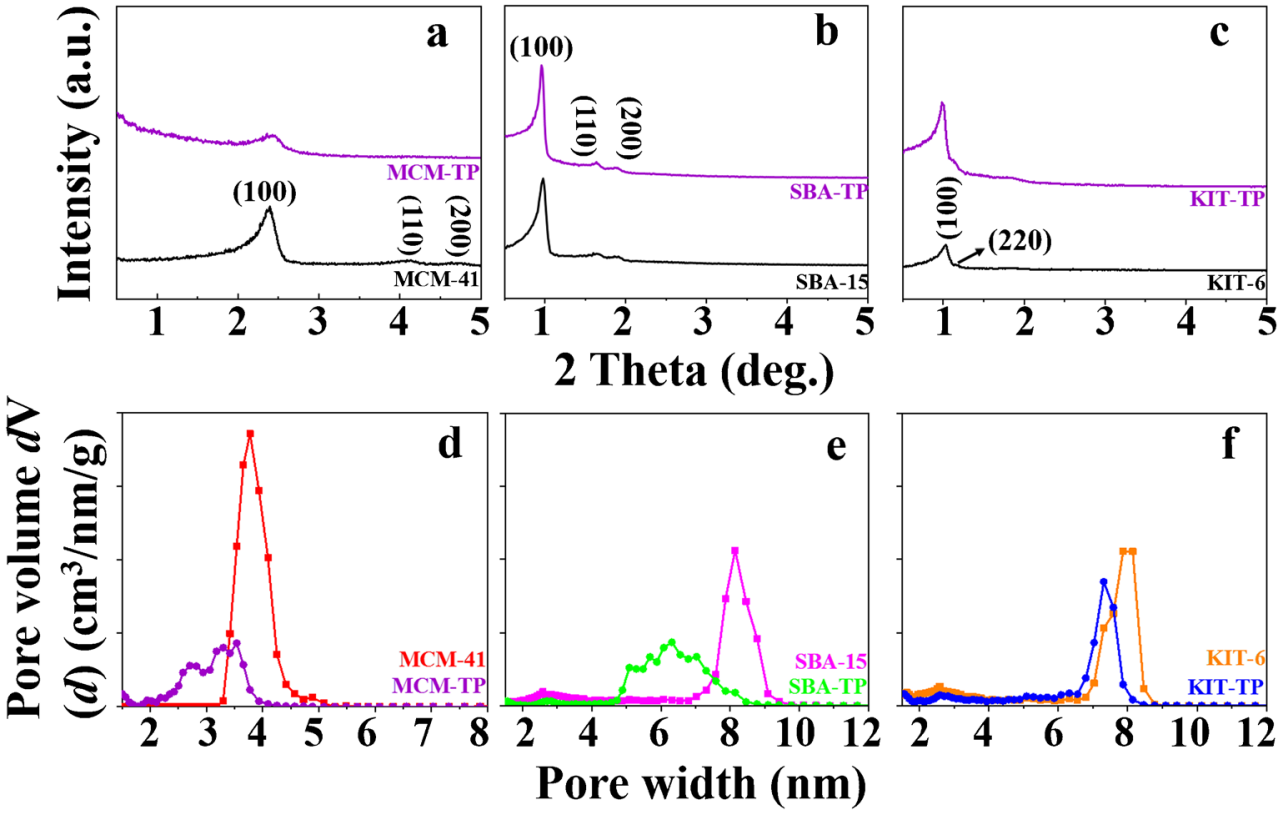

5 wt.\% of -P (Table S1), can also enhance the surface acidity of silica materials and thus promote their interaction affinity with basic analytes $[32,33]$. Therefore, comparison of the extraction performance of the aforementioned materials can help us to better understand the relation between extraction selectivity and the pore information and/or functional groups.

Upon functionalization, the three silica samples preserved their pore architectures based on the small-angle X-ray diffraction (XRD) patterns (Fig. 2a-c). For silica substrates, the weight loss was almost negligible below $600{ }^{\circ} \mathrm{C}$ (Figures S2ac). Even though the thermal stability of silica materials modified by -TP groups slightly decreased, this had no effect on their suitability for thermal desorption, which is normally carried out under $300{ }^{\circ} \mathrm{C}$. Furthermore, their particle morphologies were not altered after functionalization regardless of the spherical MCM-41, cylinder-like SBA-15 or rock-like KIT-6 morphologies (Fig. 3). The corresponding silica-coated SPME Arrows demonstrated good surface uniformity with 20 $45 \mu \mathrm{m}$ coating thickness (insets in Fig. 3a-f). Based on these characterization results, six ordered mesoporous silica materials and their corresponding SPME Arrows were successfully synthesized and fabricated, respectively.

\section{Selectivity test and selectivity mechanism of SPME Arrows}

Since six siliceous adsorbents designed provided selective and rapid extraction of LMWAAs, a standard mixture comprised of EA, DEA, TEA and aniline, representing primary, secondary, tertiary and aromatic amines, was selected to enable a comparison of the extraction affinity between self-made and commercial adsorbents. SPME Arrow with PDMS-DVB coating was the best commercial product for comparison extraction of LMWAAs after a preliminary test (Figure S3).

In order to investigate the extraction affinity of the materials to LMWAAs, SPME Arrows were operated at under $1 \%$ humidity to eliminate the interference from moistures. Siliceous coatings extracted 18.6-102.5 times, 4.8-10.8 times and 2.6-4.0 times more EA, DEA and TEA, respectively, than SPME Arrow with PDMS-DVB coating (Fig. 4a). The high extraction efficiencies observed for these six tailor-made coatings are thanks to their excellent porous structure and acidic surface. Pore size is a key factor here especially when the extraction time $(10 \mathrm{~min})$ is far from equilibrium (Fig. 6). KIT-6 and SBA-15, with similar pore sizes of $7.9 \mathrm{vs} 8.2 \mathrm{~nm}$, extracted approximately the same amounts of LMWAAs even though differences in BET specific surface area $\left(210 \mathrm{~m}^{2} \mathrm{~g}^{-1}\right)$ and pore volume $\left(0.06 \mathrm{~cm}^{3} \mathrm{~g}^{-1}\right)$ (Table S2). On the contrary, MCM-41, with a pore size of $3.8 \mathrm{~nm}$, and the highest surface area of $1182 \mathrm{~m}^{2} \mathrm{~g}^{-1}$ and similar pore volume, resulted only in $60 \%$ relative extraction compare to the other two silica materials. The pore size played an important role in the passive SPME sampling due to the random diffusion of molecules into the pore channels. On the other hand, commercial DVB had the largest pore size of $40 \mathrm{~nm}$ [34] but lowest extraction capacity of LMWAAs among the materials. This revealed that surface functional groups are also key factors for LMWAAs capturing. DVB with a more neutral and hydrophobic surface, has limited interactions with highly polar LMWAAs. However, it has good $\pi-\pi$ interactions with aromatic aniline. As well recognized silica substrates have strong interactions with basic analytes due to their surface silanol groups. The surface Lewis and Brønsted acidity was further enhanced by modification with -TP groups. However, surface grafting decreased the pore sizes and volumes of the materials. The pore 


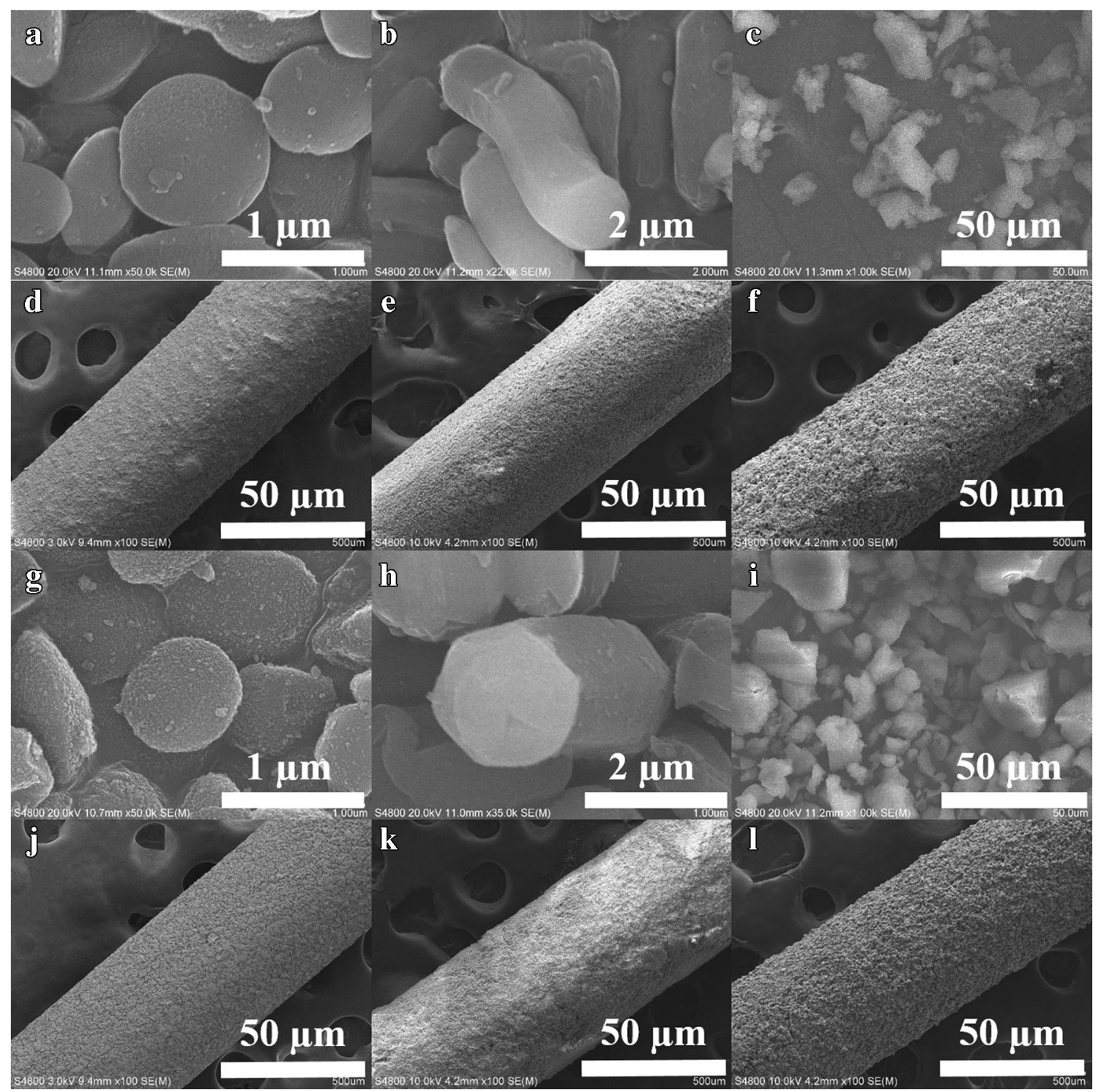

Fig. 3 Scanning electron microscopy (SEM) images of (a) MCM-41, (b) SBA-15, (c) KIT-6, (d) MCM-TP, (e) SBA-TP and (f) KIT-TP SPME Arrow coatings (scale bar, $50 \mu \mathrm{m}$ )

sizes of MCM-41, SBA-15 and KIT-6 dropped by $\sim 0.3, \sim 1.8$ and $\sim 0.5 \mathrm{~nm}$, respectively, due to the -TP modification (Fig. $2 \mathrm{~d}-\mathrm{f})$. Accordingly, this process reduced also the amount of the extracted LMWAAs by 13.9, 22.1 and $17.3 \%$, which again supported the importance of pore size in capturing small molecules (Fig. 4a).

Fig. 4 Comparison of tailor-made and commercial SPME Arrows for extraction of EA, DEA, TEA and aniline at (a) under $1 \%$ and at (b) $50 \%$ humidity

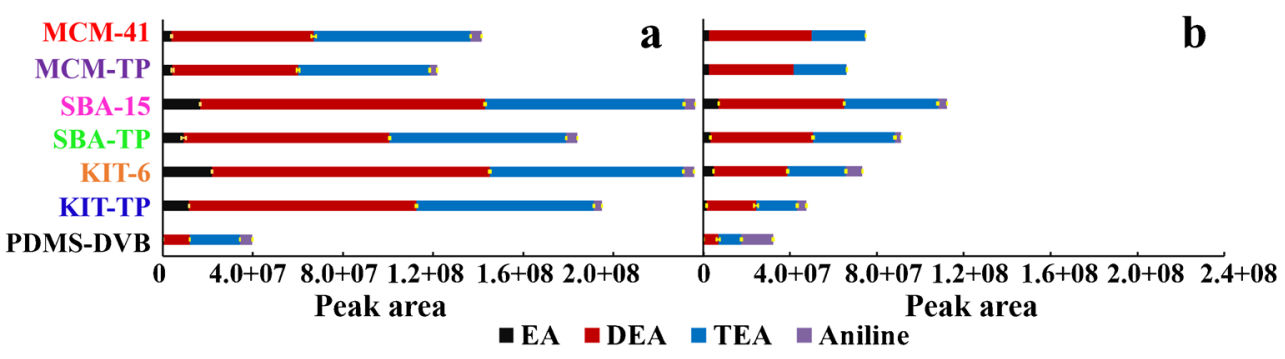


Fig. 5 a Selectivity comparison of different materials towards LMWAAs and $\mathbf{b}$ represent GCMS chromatograms of LMWAAs and other compounds in samples extracted with MCM-41, MCMTP and PDMS-DVB-SPME Arrows
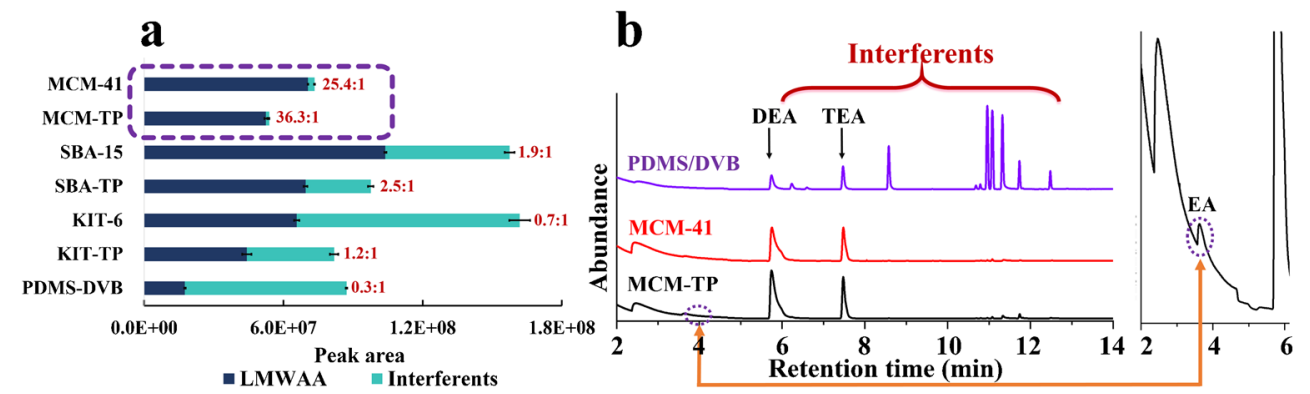

be occupied by water instead of target molecules (Figure S4). Among the silica materials, SPME Arrow with KIT-6 and KIT-TP coatings provided biggest extraction drops of 71 and $77 \%$, respectively (Fig. 4b), because of their 3D cubic pore structure and 6-8 nm pore size, which are the most accessible pores for water molecules. Interestingly, the 2D structure and $\sim 3 \mathrm{~nm}$ channels of the MCM-41 and MCM-TP materials did not allow most water molecules (Figure S4) to enter their pores, giving the smallest decrease of 44 and $45 \%$ in the extraction (Fig. 4b). The hydrophobic composition of SPME Arrow with PDMS-DVB decreased the extraction with 49\%, proving that it is easy for water molecules to enter its $40 \mathrm{~nm}$ pores (Fig. 4b). Despite of the drop in extraction performance of silica materials, their extraction efficiency to EA, DEA and TEA was still 11.6-53.3, 3.3-8.4 and 1.8-3.6 times higher than that of commercial SPME Arrow with PDMS-DVB. The results indicate that pore size is not only an important parameter for controlling LMWAAs extraction but also for preventing the interferences caused by water.

A viable extraction strategy of LMWAAs from complicated matrices does not only need a high adsorbent capacity but also selectivity, efficiency and recyclability play an important role.

The laboratory-prepared SPME Arrow silica and SPME Arrow PDMS-DVB coatings were then evaluated in terms of their ability to extract LMWAAs from a mixture containing ten representative volatile organic compounds (VOCs) ranging from 0.07 to $12.58 \mathrm{ng} \mathrm{mL} \mathrm{m}^{-1}$ of aliphatic and aromatic compounds. They included ketones, alcohols, furans, hydrocarbons and acetates (Table S3). For this mixture, SPME Arrow with MCM-41 and MCM-TP coatings gave the highest selectivity factor (defined as: total peak area ratio of LMWAAs/ten competing compounds) of 25.4:1 and 36.3:1, respectively (Fig. 5a). This can be explained by the synergism of surface functionality, pore size and pore structure of these two materials. After functionalized with the -TP group, silica materials enhanced their affinity to amines, and at the same time, their small pore size restricted the diffusion of low affinity interferents. On the other hand, due to the largest pore size and most accessible 3D pore structure, SPME Arrow with KIT-6 series therefore logically had the lowest selectivity to the amines. Although SPME Arrow with SBA-15 series had approximately the same pore size, their $2 \mathrm{D}$ channels prevents molecules to diffuse in, giving slightly higher selectivity factor while still much lower than that of SPME Arrow MCM-41 series. As an example, the chromatogram in Fig. 5b clearly shows the superiority and weak selectivity of SPME Arrow MCM-41 series and PDMS-DVB coatings, respectively. In summary, SPME Arrow MCM-41 series silica had the lowest capacity but the best selectivity to LWMAAs because of 1) their 2-4 nm pore sizes capable to capture small molecules, 2)
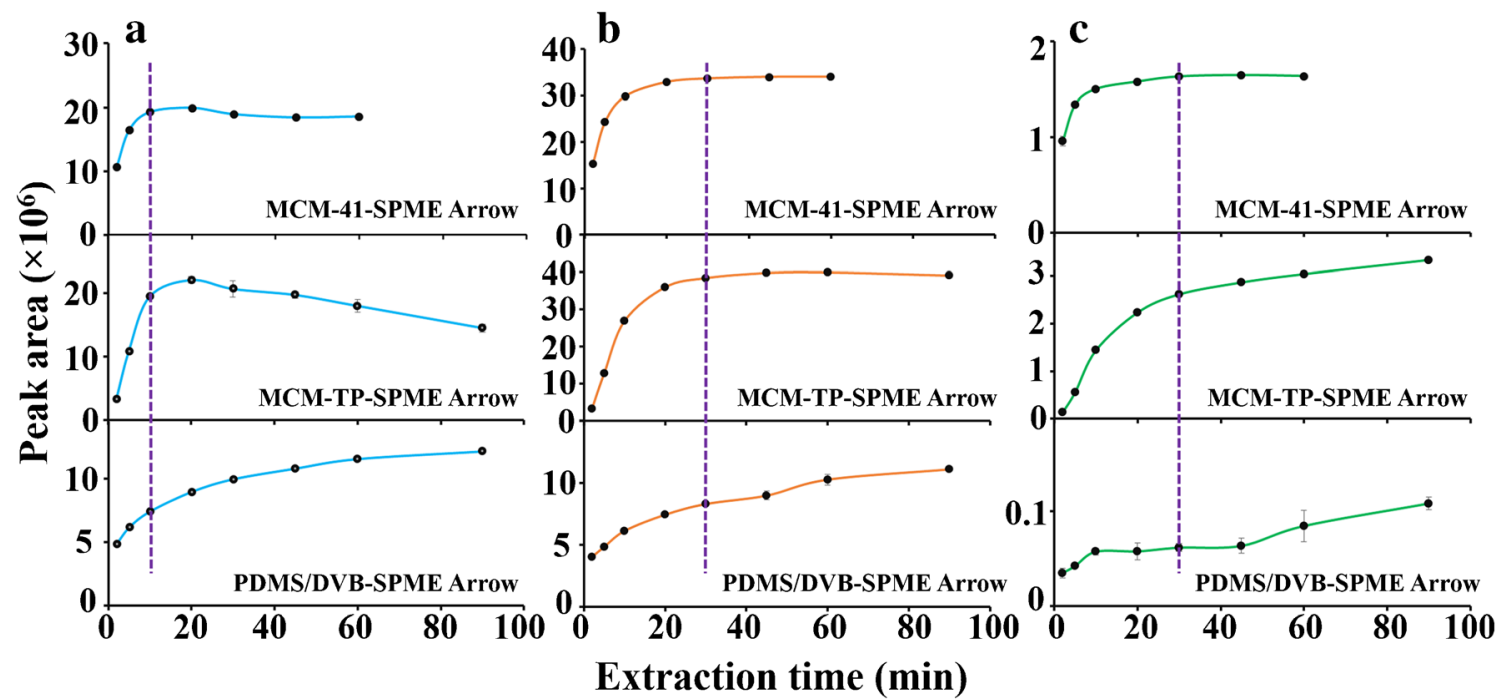

Fig. 6 Extraction kinetics of MCM-41 series and PDMS-DVB SPME Arrows for extraction of (a) TEA, (b) DEA and (c) EA 
their acidic surface that has strong affinity to LMWAAs, 3) their 2D pore channels that limit the extraction of low affinity target compounds and water molecules, and 4) their nonaromatic structure that has low affinity to aromatic compounds. Moreover, SPME Arrow with MCM-41 series coatings should also work for selective extraction of other LMWAAs, such as methylamine, dimethylamine, trimethylamine, etc.

In addition, MCM-41- and MCM-TP-SPME Arrows reached extraction equilibrium for DEA and TEA within 20 and $30 \mathrm{~min}$, respectively, which were much shorter than that of SPME Arrow with PDMS-DVB (60 min) (Fig. 6). This remarkable, fast extraction rate is attributed to the acidic surface groups which strongly trapped the analytes. However, MCM-TP-SPME Arrow extracted more EA with longer extraction time. Obviously, there is a competition in the extraction of EA and TEA by SPME Arrow with MCM-TP coating after $10 \mathrm{~min}$, indicating that the material's acidic channels prefer to interact with smaller and more polar EA. As a compromise of the extraction of EA and TEA, the extraction time should not exceed 30 min by MCM-TP-SPME Arrow.

To study the repeatability of extraction of LMWAAs by MCM-41- and MCM-TP-SPME, more than 150 extraction cycles were carried out. Since MCM-41- and MCM-TPSPME Arrows gave repeatable results, they were selected for the further studies.

\section{Optimization of mesoporous silica-coated SPME Arrow conditions}

The following parameters, which can influence the SPME Arrow performance, were optimized: (a) base addition; (b) ionic strength; (c) extraction temperature; (d) agitation speed; (e) desorption temperature and (f) desorption time. Respective data and Figures are given in the Supporting Material (Figure S5). For the optimization studies a $100 \mathrm{ng} \mathrm{mL}^{-1}$ mixture of EA, DEA and TEA was used.

\section{Extraction conditions}

The addition of base to the amine solution is crucial to neutralize and release the amines into the headspace.

During the extraction, higher temperature usually promotes the transfer of analytes from the solution to the headspace, but it can also increase the amount of water and its condensation on the SPME Arrow [35]. In addition, analytes already attached on the sorbent can be partitioned back to the headspace (equilibrium favors the gas phase) due to the high temperature. Three temperatures, 40, 50 and $60{ }^{\circ} \mathrm{C}$, were employed after considering the temperature limit of the PAL auto-sampler and water condensation. The lowest temperature, $40^{\circ} \mathrm{C}$, gave the strongest response which revealed an exothermic process. Also, the effect of different agitation speeds, 250, 500, 750, and $1000 \mathrm{rpm}$, on the recovery was tested with two SPME Arrow systems without observing any clear trend.

\section{Desorption conditions}

The amount of desorbed analytes greatly increased with higher desorption temperature and longer desorption times. However, a compromise between these two parameters was made to guarantee a long coating lifetime, while still maintaining an efficient desorption. A $60 \mathrm{~s}$ desorption step at $260{ }^{\circ} \mathrm{C}$ ensured over $95 \%$ release of amines from the two coatings tested.

In summary, the extraction and desorption conditions for the amines with MCM-41- and MCM-TP-SPME Arrows were generally the same: (a) $250 \mu \mathrm{L} 5 \mathrm{M} \mathrm{KOH}$; (b) $2 \mathrm{~g}$ $\mathrm{NaCl}$; (c) $40{ }^{\circ} \mathrm{C}$ extraction temperature; (d) $250 \mathrm{rpm}$ agitation speed; (e) $260{ }^{\circ} \mathrm{C}$ desorption temperature and (f) $60 \mathrm{~s}$ desorption time. Extraction time was exceptionally 20 and $30 \mathrm{~min}$ for MCM-41- and MCM-TP-SPME Arrows, respectively.

\section{Method validation}

The performance of the MCM-41- and MCM-TP-SPME Arrows for amine analysis under the optimized conditions (calibration plots, limits of detection (LODs), limits of quantitation (LOQs), coefficient of determination and repeatability) is shown in Table S4. Triplicate measurements at eight concentration levels were processed for the calibration plots. LODs and LOQs were determined as concentrations giving the signal-to-noise ratio of three and ten, respectively. In general, MCM-41-SPME Arrow-GC-MS exhibited slightly lower LODs and LOQs than that of MCM-TP-SPME Arrow-GCMS because of its higher extraction capacity. Both materials showed linearity with good correlation coefficients $\left(R^{2}\right)$, which were higher than 0.9955 , and good repeatability (RSD $<20.1 \%$ ). The method sensitivity for TEA determination in this study was two magnitudes higher than that in our previous work [14]. On the other hand, MCM-41 and MCMTP-SPME Arrow-GC-MS methods demonstrated advantages over to other SPME-based methods for LMWAA determinations in terms of selectivity, sensitivity, linear range or repeatability (Table S5).

\section{Sample analysis}

\section{Mushroom samples}

In the six mushroom samples, DEA and TEA were detected and their concentrations ranged from $181.7 \pm 6.1$ to $373.3 \pm$ 19.2 and $18.4 \pm 2.1$ to $41.8 \pm 0.7 \mathrm{ng} \mathrm{g}^{-1}$ by MCM-41-SPME Arrow and $174.9 \pm 1.0$ to $375.7 \pm 12.4$ and $19.5 \pm 2.5$ to 38.5 $\pm 2.6 \mathrm{ng} \mathrm{g}^{-1}$ by MCM-TP-SPME Arrow, respectively. The results proved also that there was a good correlation between 
Table 1 Analytical results for mushroom samples by MCM-41- and MCM-TP-SPME Arrow extraction and GC-MS analysis

\begin{tabular}{|c|c|c|c|c|c|c|}
\hline \multirow[t]{2}{*}{ Mushroom } & \multicolumn{3}{|c|}{ MCM-41-SPME Arrow } & \multicolumn{3}{|c|}{ MCM-TP-SPME Arrow } \\
\hline & $\mathrm{EA}\left(\operatorname{ng~g}^{-1}\right)$ & DEA $\left(\operatorname{ng~g}^{-1}\right)$ & TEA $\left(n g g^{-1}\right)$ & $\mathrm{EA}\left(n \mathrm{ng}^{-1}\right)$ & $\operatorname{DEA}\left(\mathrm{ng} \mathrm{g}^{-1}\right)$ & TEA (ng g $\left.{ }^{-1}\right)$ \\
\hline$\# 1$ & ND & $288.9 \pm 4.3$ & $23.5 \pm 0.8$ & ND & $308.4 \pm 12.9$ & $30.4 \pm 2.3$ \\
\hline$\# 2$ & ND & $334.5 \pm 6.8$ & $34.6 \pm 4.2$ & ND & $354.6 \pm 12.3$ & $34.3 \pm 0.4$ \\
\hline \#3 & ND & $282.3 \pm 7.1$ & $41.8 \pm 0.7$ & ND & $291.9 \pm 23.1$ & $38.5 \pm 2.6$ \\
\hline$\# 4$ & ND & $260.0 \pm 15.1$ & $28.7 \pm 0.7$ & ND & $250.5 \pm 3.7$ & $28.8 \pm 0.8$ \\
\hline$\# 5$ & ND & $181.7 \pm 6.1$ & $18.4 \pm 2.1$ & ND & $174.9 \pm 1.0$ & $19.5 \pm 2.5$ \\
\hline$\# 6$ & ND & $373.3 \pm 19.2$ & $24.1 \pm 1.7$ & ND & $375.7 \pm 12.4$ & $27.1 \pm 1.6$ \\
\hline$\# 2$ & $\begin{array}{l}\text { Recovery } \\
\text { (RSD) \% }\end{array}$ & $\begin{array}{l}\text { Recovery } \\
\text { (RSD) \% }\end{array}$ & $\begin{array}{l}\text { Recovery } \\
\text { (RSD) \% }\end{array}$ & $\begin{array}{l}\text { Recovery } \\
\text { (RSD) \% }\end{array}$ & $\begin{array}{l}\text { Recovery } \\
\text { (RSD) \% }\end{array}$ & Recovery (RSD) \% \\
\hline $20 \mathrm{ng} \mathrm{g}^{-1}$ & $81.1(7.9)$ & $93.6(7.5)$ & $83.3(4.3)$ & $75.9(2.1)$ & $97.1(14.5)$ & $87.8(4.6)$ \\
\hline $50 \mathrm{ng} \mathrm{g}^{-1}$ & $91.1(3.8)$ & $95.6(6.5)$ & $89.6(12.0)$ & $86.6(11.5)$ & $89.9(1.7)$ & $95.4(4.3)$ \\
\hline $100 \mathrm{ng} \mathrm{g}^{-1}$ & $104.1(2.6)$ & $91.6(1.5)$ & $102.2(8.9)$ & $96.6(7.5)$ & $86.6(5.8)$ & $105.1(1.0)$ \\
\hline
\end{tabular}

two different self-made SPME Arrows. Mushroom sample \#2 was used for recovery test and it was spiked with three amines at 20, 50 and $100 \mathrm{ng} \mathrm{g}^{-1}$ levels. Both MCM-41- and MCMTP-SPME Arrows gave good recoveries $(\geq 75.9 \%)$ and repeatability $(\leq 14.5 \%)$ (Table 1$)$.

\section{Atmospheric air samples}

MCM-TP-SPME Arrow was used for the analysis of atmospheric air samples. DEA concentration ranged between $6.2-103.7 \mathrm{ng} \mathrm{m}^{-3}$ in the first three days and TEA concentration ranged between $0.1-8.6 \mathrm{ng} \mathrm{m}^{-3}$ in four days (Figure S6). The results revealed that there were amine emissions in the boreal forest even at low temperatures $\left(-1.4-3.3{ }^{\circ} \mathrm{C}\right)$ and the snow in the last two days decreased their levels significantly. Amine concentrations in this study were slightly higher but still in the same order of magnitude as those reported in the literature $[4,5]$.

\section{Urine sample}

The urine sample was analyzed by GC-MS utilizing MCM-41-, MCM-TP- and PDMS-DVB-SPME Arrow systems (Fig. 7). Laboratory-made SPME Arrows gave much more intense amine peaks and clearer baselines compared to the commercial PDMS-DVB-SPME Arrow. In the sample, ammonia, dimethylamine (DMA) +EA, DEA and TEA were identified with all laboratory-made SPME Arrows mentioned above.

\section{Conclusions}

In this study, SPME Arrows including different self-made mesoporous silica coatings were utilized to capture rapidly targeted LMWAAs from various sample matrices. SPME Arrows with MCM-41 and MCM-TP coatings having $2 \mathrm{D}$ channels and a pore size of $<4 \mathrm{~nm}$ gave superior selectivity towards LMWAAs when compared to another self-made SPME Arrow silica coatings SBA-15 and KIT6 , and to several commercial materials. The success of the extraction and especially the extraction selectivity by using different SPME Arrow coatings were studied by clarifying two main properties: 1) size exclusion, in terms of pore size and pore structure, and 2) acid-base interaction, by increasing silica surface acidity by functionalization with -TP groups resulting in more acidic Lewis and Brønsted sites. Furthermore, optimal laboratory-made SPME Arrows demonstrated fast extraction kinetics, good reusability and convenience for the
Fig. 7 GC-MS chromatograms of the urine samples after extraction by MCM-41, MCM-TP and PDMS-DVB coated SPME Arrows

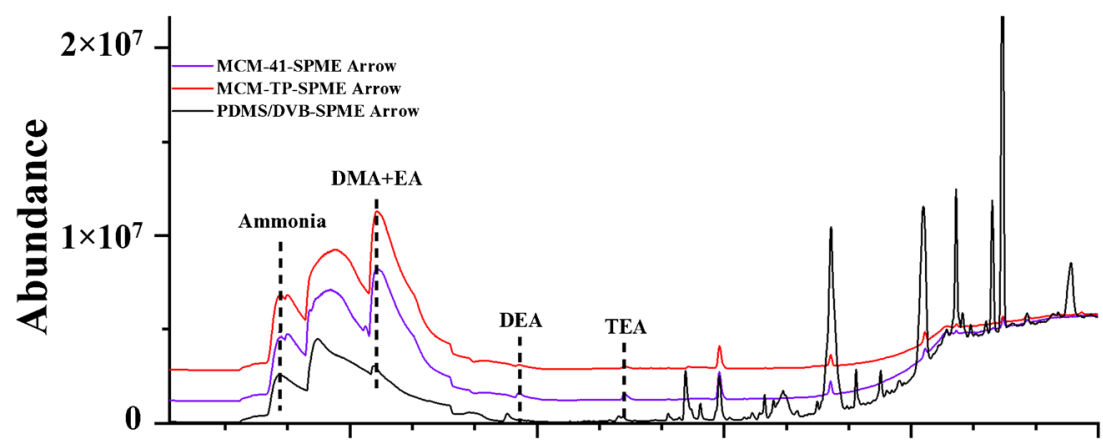


development of analytical methods. The SPME Arrow GC-MS methods at optimal conditions were validated and further applied to the identification and quantitation of LMWAAs in mushroom, atmospheric air and urine samples. The results achieved indicated that functionalized mesoporous silica was suitable for the extraction and enrichment of LMWAAs in different sample matrices. With a careful selection of silica substrate and functional groups, new materials allow to capture selectively many other small and polar VOCs from liquid or gaseous samples. Furthermore, an exhaustive sampling technique, such as in-tube extraction and needle trap extraction, can improve the method sensitivity and the quantitation further.

Acknowledgements Financial support was provided by China Scholarship Council (H.L. grant no. 201508330310) and the Finnish Centre of Excellence in Atmospheric Science-From Molecular and Biological Processes to the Global Climate (grant no. 307331). CTC Analytics AG (Zwingen, Switzerland) and BGB Analytik AG (Zurich, Switzerland) are thanked for the cooperation.

Funding Information Open access funding provided by University of Helsinki including Helsinki University Central Hospital.

Compliance with ethical standards The author(s) declare that they have no competing interests.

Open Access This article is distributed under the terms of the Creative Commons Attribution 4.0 International License (http:// creativecommons.org/licenses/by/4.0/), which permits unrestricted use, distribution, and reproduction in any medium, provided you give appropriate credit to the original author(s) and the source, provide a link to the Creative Commons license, and indicate if changes were made.

\section{References}

1. Ge X, Wexler AS, Clegg SL (2011) Atmospheric amines-part I. a review. Atmos Environ 45(3):524-546

2. Murphy S, Sorooshian A, Kroll J, Ng N, Chhabra P, Tong C, Surratt J, Knipping E, Flagan R, Seinfeld J (2007) Secondary aerosol formation from atmospheric reactions of aliphatic amines. Atmospheric Chem Phys 7(9):2313-2337

3. Smith JN, Barsanti KC, Friedli HR, Ehn M, Kulmala M, Collins DR, Scheckman JH, Williams BJ, McMurry PH (2010) Observations of aminium salts in atmospheric nanoparticles and possible climatic implications. Proc Natl Acad Sci 107(15):6634 6639

4. Kieloaho A-J, Hellén H, Hakola H, Manninen HE, Nieminen T, Kulmala M, Pihlatie M (2013) Gas-phase alkylamines in a boreal scots pine forest air. Atmos Environ 80:369-377

5. Hellén H, Hakola H, Reissell A, Ruuskanen T (2004) Carbonyl compounds in boreal coniferous forest air in Hyytiälä, southern Finland. Atmos Chem Phys 4(7):1771-1780

6. Wolrath H, Borén H, Hallén A, Forsum U (2002) Trimethylamine content in vaginal secretion and its relation to bacterial vaginosis. Apmis 110(11):819-824
7. Zeisel SH, Wishnok JS, Blusztajn J (1983) Formation of methylamines from ingested choline and lecithin. J Pharmacol Exp Ther 225(2):320-324

8. Lundh T, Åkesson B (1993) Gas chromatographic determination of primary and secondary low-molecular-mass aliphatic amines in urine using derivatization with isobutyl chloroformate. J Chromatogr B Biomed Sci 617(2):191-196

9. Bain MA, Faull R, Fornasini G, Milne RW, Evans AM (2006) Accumulation of trimethylamine and trimethylamine-N-oxide in end-stage renal disease patients undergoing haemodialysis. Nephrol Dial Transplant 21(5):1300-1304

10. Lynch DW, Moorman WJ, Stober P, Lewis TR, Iverson WO (1986) Subchronic inhalation of diethylamine vapor in Fischer-344 rats: organ system toxicity. Toxicol Sci 6(3):559-565

11. Feijó Barreira LM, Duporté G, Rönkkö T, Parshintsev J, Hartonen K, Hyrsky L, Heikkinen E, Jussila M, Kulmala M, Riekkola M-L (2018) Field measurements of biogenic volatile organic compounds in the atmosphere using solid-phase microextraction arrow. Atmos Meas Tech 11(2):881-893

12. Helin A, Rönkkö T, Parshintsev J, Hartonen K, Schilling B, Läubli T, Riekkola M-L (2015) Solid phase microextraction arrow for the sampling of volatile amines in wastewater and atmosphere. J Chromatogr A 1426:56-63

13. Kremser A, Jochmann MA, Schmidt TC (2016) PAL SPME arrowevaluation of a novel solid-phase microextraction device for freely dissolved PAHs in water. Anal Bioanal Chem 408(3):943-952

14. Lan H, Rönkkö T, Parshintsev J, Hartonen K, Gan N, Sakeye M, Sarfraz J, Riekkola M-L (2017) Modified zeolitic imidazolate framework- 8 as solid-phase microextraction arrow coating for sampling of amines in wastewater and food samples followed by gas chromatography-mass spectrometry. J Chromatogr A 1486:76-85

15. Lan H, Salmi LD, Rönkkö T, Parshintsev J, Jussila M, Hartonen K, Kemell M, Riekkola M-L (2018) Integrated atomic layer deposition and chemical vapor reaction for the preparation of metal organic framework coatings for solid-phase microextraction arrow. Anal Chim Acta 1024:93-100

16. Rocío-Bautista P, Pacheco-Fernández I, Pasán J, Pino V (2016) Are metal-organic frameworks able to provide a new generation of solid-phase microextraction coatings?-a review. Anal Chim Acta 939:26-41

17. Wang Y, Rui M, Lu G (2018) Recent applications of metal-organic frameworks in sample pretreatment. J Sep Sci 41(1):180-194

18. Ismail AF, David L (2001) A review on the latest development of carbon membranes for gas separation. J Membr Sci 193(1):1-18

19. Maya F, Cabello CP, Ghani M, Palomino GT, Cerda V (2018) Emerging materials for sample preparation. J Sep Sci 41(1):262287

20. Mohamed AR, Mohammadi M, Darzi GN (2010) Preparation of carbon molecular sieve from lignocellulosic biomass: a review. Renew Sust Energ Rev 14(6):1591-1599

21. Sethupathi S, Bashir MJK, Akbar ZA, Mohamed AR (2015) Biomass-based palm shell activated carbon and palm shell carbon molecular sieve as gas separation adsorbents. Waste Manag Res 33(4):303-312

22. Yao JZ, Sun NR, Deng CH (2018) Recent advances in mesoporous materials for sample preparation in proteomics research. TrAC Trends Anal Chem 99:88-100

23. Kleitz F, Choi SH, Ryoo R (2003) Cubic Ia 3 d large mesoporous silica: synthesis and replication to platinum nanowires, carbon nanorods and carbon nanotubes. Chem Commun (17):2136-2137

24. Zhao D, Huo Q, Feng J, Chmelka BF, Stucky GD (1998) Nonionic triblock and star diblock copolymer and oligomeric surfactant syntheses of highly ordered, hydrothermally stable, mesoporous silica structures. J Am Chem Soc 120(24):6024-6036

25. Cai Q, Lin W-Y, Xiao F-S, Pang W-Q, Chen X-H, Zou B-S (1999) The preparation of highly ordered MCM-41 with extremely low 
surfactant concentration. Microporous Mesoporous Mater 32(1-2): $1-15$

26. Zhang J, Ma Z, Jiao J, Yin H, Yan W, Hagaman EW, Yu J, Dai S (2009) Layer-by-layer grafting of titanium phosphate onto mesoporous silica SBA-15 surfaces: synthesis, characterization, and applications. Langmuir 25(21):12541-12549

27. Hari P, Kulmala M (2005) Station for measuring ecosystematmosphere relations (SMEAR II). Boreal Environ Res 10(5): 315-322

28. Kruk M, Jaroniec M, Sayari A (1997) Application of large pore MCM-41 molecular sieves to improve pore size analysis using nitrogen adsorption measurements. Langmuir 13(23):6267-6273

29. Zhao D, Feng J, Huo Q, Melosh N, Fredrickson GH, Chmelka BF, Stucky GD (1998) Triblock copolymer syntheses of mesoporous silica with periodic 50 to 300 angstrom pores. Science 289(5350): $548-552$

30. Kleitz F, Berube F, Guillet-Nicolas R, Yang C-M, Thommes M (2010) Probing adsorption, pore condensation, and hysteresis behavior of pure fluids in three-dimensional cubic mesoporous KIT-6 silica. J Phys Chem C 114(20):9344-9355

31. Kovalchuk T-V, Sfihi H, Korchev A-S, Kovalenko A-S, Il'in V-G, Zaitsev V-N, Fraissard J (2005) Synthesis, structure, and acidic properties of MCM-41 functionalized with phosphate and titanium phosphate groups. J Phys Chem B 109(29):13948-13956
32. Hua W, Yue Y, Gao Z (2001) Acidity enhancement of SBA mesoporous molecular sieve by modification with $\mathrm{SO}_{4}{ }^{2-} / \mathrm{ZrO}_{2}$. J Mol Catal A Chem 170(1-2):195-202

33. Ramanathan A, Maheswari R, Barich DH, Subramaniam BJM (2014) Niobium incorporated mesoporous silicate, Nb-KIT-6: synthesis and characterization. Microporous Mesoporous Mater 190: 240-247

34. Grandy JJ, Singh V, Lashgari M, Gauthier M, Pawliszyn J (2018) Development of a hydrophilic lipophilic balanced thin film solid phase microextraction device for balanced determination of volatile organic compounds. Anal Chem 90(23):14072-14080

35. Hartonen K, Helin A, Parshintsev J, Riekkola M-L (2019) Problems caused by moisture in gas chromatographic analysis of headspace SPME samples of short-chain amines. Chromatographia 82(1):307-316

Publisher's note Springer Nature remains neutral with regard to jurisdictional claims in published maps and institutional affiliations. 